\title{
A NOTE ON THE BOARDMAN THEOREM
}

\author{
ZHI LÜ \\ Department of Applied Mathematics \\ Tsinghua University \\ Beijing, 100084, P.R. CHINA \\ and \\ CHUN-LIAN ZHOU \\ Department of Mathematics \\ Armored Force Engineering Institute \\ Beijing, 100072, P.R. CHINA
}

(Received June 18, 1996)

\begin{abstract}
Let $\left(T, M^{n}\right)$ be a smooth involution on a closed $n$-dimensional manifold with $n \leq \frac{5}{2} \operatorname{dim} F$. This paper gives three necessary and sufficient conditions that $\left(T, M^{n}\right)$ is a bounding involution.
\end{abstract}

KEY WORDS AND PHRASES: Involution, fixed point set, projective space bundle. 1991 AMS SUBJECT CLASSIFICATION CODE: Primary 57R85.

\section{INTRODUCTION}

Let $\left(T, M^{n}\right)$ be a smooth involution on a closed $n$-dimensional manifold. Let $F$ denote the set of fixed points of $\left(T, M^{n}\right)$. This fixed point set is the finite, disjoint union of closed submanifolds of $M^{n}$ (see [1]). By $\operatorname{dim} F$ we mean the dimension of the highest dimensional nonempty component of $F$. In Conner's book [1], we know the following important result due to Boardman.

THEOREM (Boardman). If $\left(T, M^{n}\right)$ be an involution on a closed manifold $M^{n}$ for which $n>\frac{5}{2} \operatorname{dim} F$, then $\left(T, M^{n}\right)$ bounds.

But if we change the condition $n>\frac{5}{2} \operatorname{dim} F$ into $n \leq \frac{5}{2} \operatorname{dim} F$ in the Boardman Theorem, then generally the result doesn't hold. An example can be seen in [3]. In this paper, we focus our attention on the case in which $n \leq \frac{5}{2} \operatorname{dim} F$, and give three necessary and sufficient conditions that $\left(T, M^{n}\right)$ with $n \leq \frac{5}{2} \operatorname{dim} F$ bounds.

Throughout this paper, the coefficient group is $Z_{2} .\{N\}$ denote the unoriented bordism class of closed smooth manifold $N$, and $\left\{\left(T, M^{n}\right)\right\}$ the unoriented equivalent bordism class of the involution $\left(T, M^{n}\right) . \quad R P(\xi)$ denote the total space of the projective space bundle induced by vector bundle $\xi \rightarrow V^{m}$.

\section{THE MAIN RESULTS}

Recall that the unoriented bordism class of an involution is uniquely determined by the bordism class of the normal bundle to the fixed point set ([1]). We denote by $M O_{m}(B O(p))$ the unoriented bordism group of real $p$-dimensional plane bundle over closed smooth $m$-dimensional manifolds. We have

THEOREM A. Let $\mu \rightarrow F=\sqcup_{k}\left(\mu^{k} \rightarrow F^{n-k}\right)$ be the normal bundle to the fixed point set of an involution $\left(T, M^{n}\right)$ with $n \leq \frac{5}{2} \operatorname{dim} F$. Then a necessary and sufficient condition that $\left\{\left(T, M^{n}\right)\right\}=0$ is 
that $\mu \oplus\left(\left[\frac{5}{2} \operatorname{dim} F-n\right]+1\right) R \rightarrow F$ still be the normal bundle to the fixed point set of a certain involution. (Here $\left[\frac{5}{2} \operatorname{dim} F-n\right]$ denote the integer part of $\frac{5}{2} \operatorname{dim} F-n$.)

PROOF. For convenience, let $a=\left[\frac{5}{2} \operatorname{dim} F-n\right]$. Suppose

$$
\mu \oplus(a+1) R \rightarrow F=\sqcup_{k}\left(\mu^{k} \oplus(a+a) R \rightarrow F^{n-k}\right)
$$

still be the normal bundle to the fixed point set of a certain involution, denoted by $\left(T^{\prime}, V^{n+a+1}\right)$. As in [2], since $n+a+1=n+\left[\frac{5}{2} \operatorname{dim} F-n\right]+1>\frac{5}{2} \operatorname{dim} F$, by the Boardman Theorem, it follows that $\left\{\left(T^{\prime}, V^{n+a+1}\right)\right\}=0$, and thus $\left\{\mu^{k} \oplus(a+1) R \rightarrow F^{n-k}\right\}=0$ in $M O_{n-k}(B O(k+a+1))$ for each $k$. Furthermore, for each $k$, since

$$
\oplus(a+1) R: M O_{n-k}(B O(k)) \rightarrow M O_{n-k}(B O(k+a+1))
$$

is monomorphism, we conclude that for each $k$,

$$
\left\{\mu^{k} \rightarrow F^{n-k}\right\}=0
$$

in $M O_{n-k}(B O(k))$. Hence we have $\left\{\left(T, M^{n}\right)\right\}=0$.

Conversely, if $\left\{\left(T, M^{n}\right)\right\}=0$, then it immediately follows that $\left\{\mu^{k} \rightarrow F^{n-k}\right\}=0$ in $M O_{n-k}(B O(k))$ for each $k$, and thus $\left\{\mu^{k} \oplus i R \rightarrow F^{n-k}\right\}=0$ in $M O_{n-k}(B O(k+i))$ for each $k$ and all $i$. This means that for all $i$,

$$
\mu \oplus i R \rightarrow F=\sqcup_{k}\left(\mu^{k} \oplus i R \rightarrow F^{n-k}\right)
$$

be the fixed data of bounding involution. This completes the proof.

Now we consider other two necessary and sufficient conditions that involution $\left(T, M^{n}\right)$ with $n \leq \frac{5}{2} \operatorname{dim} F$ bounds.

Given any involution $\left(T, M^{n}\right)$, as defined in [1], let $A^{1}\left(M^{n}\right)$ denote a $(n+1)$-dimensional manifold formed from the product $S^{1} \times M^{n}$ of the 1-sphere with $M^{n}$ by identifying $(z, x)$ with $(-z, T x)$, and an involution $T_{1}$ on $A^{1}\left(M^{n}\right)$ be induced by $(z, x) \rightarrow(\bar{z}, x)$. Let $\left(T_{0}, A^{0}\left(M^{n}\right)\right)=\left(T, M^{n}\right)$, and $\left(T_{r}, A^{r}\left(M^{n}\right)\right)$ the $r$-th iterate of $\left(T, M^{n}\right)$. Thus a sequence of involutions $\left(T_{r}, A^{r}\left(M^{n}\right)\right)$ are constructed. In particular, we also know from [1] that the normal bundle to the fixed point set of $\left(T_{r}, A^{r}\left(M^{n}\right)\right)$ is

$$
\sqcup_{k}\left(\mu^{k} \oplus r R \rightarrow F^{n-k}\right) \sqcup\left(\sqcup_{j=0}^{r-1}(r-j) R \rightarrow A^{J}\left(M^{n}\right)\right)
$$

where $\mu \rightarrow F=\sqcup_{k}\left(\mu^{k} \rightarrow F^{n-k}\right)$ is the original normal bundle to $F$ in $M^{n}$.

LEMMA 2.1. If $\mu \rightarrow F=\sqcup_{k}\left(\mu^{k} \rightarrow F^{n-k}\right)$ be the fixed data of an involution $\left(T, M^{n}\right)$, then a necessary and sufficient condition that $\mu \oplus r R \rightarrow F$ still be the fixed data of a certain involution is that $\left\{A^{J}\left(M^{n}\right)\right\}=0$ for all $j \leq r-1$.

PROOF. First, since $\sqcup_{k}\left(\mu^{k} \oplus r R \rightarrow F^{n-k}\right) \sqcup\left(\sqcup_{j=0}^{r-1}(r-j) R \rightarrow A^{j}\left(M^{n}\right)\right)$ is the fixed data of $\left(T_{r}, A^{r}\left(M^{n}\right)\right)$, by [2, p. 328, Section 11, Proposition], we have

$$
\sum_{k} \frac{f(1+y, z)}{\Pi(1+y)}\left[F^{n-k}\right]+\sum_{j=0}^{r-1} \frac{f(1+y, z)}{\pi(1+y)}\left[A^{J}\left(M^{n}\right)\right]=0
$$

for all the symmetric polynomial functions $f\left(x_{1}, \cdots, x_{n+r}\right)$ such that $\operatorname{deg} f<n+r$.

Next, according to [2, p. 328, Section 11, Proposition] again, from (2.1) it is easy to see that $\mu \oplus r R \rightarrow F$ be the fixed data of a certain involution if and only if $\cup_{j=0}^{r-1}(r-j) R \rightarrow A^{\jmath}\left(M^{n}\right)$ also be the fixed data of a certain involution.

If $\left\{A^{J}\left(M^{n}\right)\right\}=0$ for $0 \leq j \leq r-1$, then $(r-j) R \rightarrow A^{J}\left(M^{n}\right)$ with trivial bundle bounds for $0 \leq j \leq r-1$. Thus we have 


$$
\sum_{\jmath=0}^{r-1} \frac{f(1+y, z)}{\Pi(1+y)}\left[A^{\jmath}\left(M^{n}\right)\right]=0
$$

for $\operatorname{deg} f<n+r$. This means that $\sqcup_{j=0}^{r-1}(r-j) R \rightarrow A^{j}\left(M^{n}\right)$ be the fixed data of a certain involution

On the other hand, suppose $\sqcup_{j=0}^{r-1}(r-j) R \rightarrow A^{J}\left(M^{n}\right)$ be the fixed data of a certain involution Since $\operatorname{dim} A^{\jmath}\left(M^{n}\right)=n+j<n+r$ and all the normal Whitney classes of $(r-j) R \rightarrow A^{\mathcal{J}}\left(M^{n}\right)$ vanish in position dimension for each $j \leq r-1$, by [1, Theorem 23.1], it immediately follows that $\left\{A^{\mathcal{J}}\left(M^{n}\right)\right\}=0$ for $0 \leq j \leq r-1$. Hence the result holds.

LEMMA 2.2. Let $\mu \rightarrow F$ be the fixed data of an involution $\left(T, M^{n}\right)$. Then $\left\{A^{\mathcal{J}}\left(M^{n}\right)\right\}=0$ for all $j \geq r$ if and only if $\{R P(\mu \oplus(j+1) R)\}=0$ for all $j \leq r$.

PROOF. According to [1, Lemma 25.6], we have

$$
\left\{A^{r}\left(M^{n}\right)\right\}=\{R P(\mu \oplus(r+1) R)\}+\sum_{j=0}^{r-1}\{R P(r-j)\}\left\{A^{\jmath}\left(M^{n}\right)\right\} .
$$

From (2.2), using induction on $r$, it is easy to show that the Lemma holds.

Combining Theorem A and Lemmas 2.1 and 2.2, we at once have

THEOREM B. Let $\mu \rightarrow F$ be the fixed data of an involution $\left(T, M^{n}\right)$ with $n \leq \frac{5}{2} \operatorname{dim} F$. Then ' $\left\{\left(T, M^{n}\right)\right\}=0$ if and only if either

(i) $\left\{A^{J}\left(M^{n}\right)\right\}=0$ for all $j \leq\left[\frac{5}{2} \operatorname{dim} F-n\right]$ or

(ii) $\{R P(\mu \oplus(j+1) R)\}=0$ for all $j \leq\left[\frac{5}{2} \operatorname{dim} F-n\right]$.

In [2], Kosniowski and Stong proved that

THEOREM C. If $\left(T, M^{n}\right)$ be a smooth involution with fixed point set of constant dimension and $n>2 \operatorname{dim} F$, then $\left\{\left(T, M^{n}\right)\right\}=0$.

As before, from Theorem $C$, we also can obtain

THEOREM D. Let $\left(T, M^{n}\right)$ be a smooth involution with fixed point set $F^{k}$ of constant dimension $k$ and $\mu^{n-k} \rightarrow F^{k}$ be the fixed data of $\left(T, M^{n}\right)$. If $n \leq 2 k$, then there exist the following three necessary and sufficient conditions that $\left\{\left(T, M^{n}\right)\right\}=0$

(i) $\left\{R P\left(\mu^{n-k} \oplus(i+1) R\right)\right\}=0$ for all $i \leq 2 k-n$,

(ii) $\left\{A^{2}\left(M^{n}\right)\right\}=0$ for all $i \leq 2 k-n$,

(iii) $\mu^{n-k} \oplus(2 k-n+1) R \rightarrow F^{k}$ still be the fixed data of a certain involution.

\section{THE NORMAL BUNDLE TO THE FIXED POINT SET}

From Lemma 2.1, an interesting result can easily be obtained, i.e., that is the following

PROPOSITION 3.1. Let $\mu \rightarrow F$ be the fixed data of an involution $\left(T, M^{n}\right)$. If $\mu \oplus r R \rightarrow F$ be the fixed data of a certain involution, then $\mu \oplus i R \rightarrow F$ also be the fixed data of a certain involution for each $i<r$.

Proposition 3.1 can be used well in discussing the existence of the involutions fixing the disjoint union of the 3-projective space with the Dold manifold (see [4]). Here we give a simple example of using Proposition 3.1.

EXAMPLE 1. We define an involution $T$ on $R P(3)$ by

$$
T:\left(x_{0}, x_{1}, x_{2}, x_{3}\right) \rightarrow\left(-x_{0}, x_{1}, x_{2}, x_{3}\right) .
$$

Then the fixed point set of $(T, R P(3))$ is $F=* \sqcup R P(2)$, and it is easy to see that the normal bundle to $F=* \sqcup R P(2)$ in $R P(3)$ is

$$
\mu \rightarrow F=(3 R \rightarrow *) \cup(\lambda \rightarrow R P(2))
$$


where $\lambda \rightarrow R P(2)$ is a canonical line bundle over $R P(2)$, and * denotes a point. From [3], we know that

$$
(5 R \rightarrow *) \sqcup(\lambda \oplus 2 R \rightarrow R P(2))
$$

still be the fixed data of an involution $\left(T^{\prime}, M^{5}\right)$ and $\left\{M^{5}\right\}=\{R P(2,0,0,0)\}$. By Proposition 31 , we at once know that

$$
(4 R \rightarrow *) \sqcup(\lambda \oplus R \rightarrow R P(2))
$$

must be the fixed data of a certain involution. Of course, we also know that $\{R P(3)\}=0$, $\left\{A^{1}(R P(3))\right\}=0,\left\{A^{2}(R P(3))\right\}=\{R P(2,0,0,0)\} \neq 0$, and thus

$$
(6 R \rightarrow *) \sqcup(\lambda \oplus 3 R \rightarrow R P(2))
$$

must not be the fixed data of a certain involution and $\{(T, R P(3))\} \neq 0$.

REMARK. Example 1 tells us that in Theorem A, if $\mu \oplus\left(\left[\frac{5}{2} \operatorname{dim} f-n\right]\right) R \rightarrow F$ be the fixed data of a certain involution, but $\mu \oplus\left(\left[\frac{5}{2} \operatorname{dim} F-n\right]+1\right) R \rightarrow F$ be not the fixed data of a certain involution, then the result fails. This means that in Theorem $\mathrm{B},\left[\frac{5}{2} \operatorname{dim} F-n\right]$ is exactly the least upper bound of all $j$ such that $\left\{A^{J}\left(M^{n}\right)\right\}=0$ and $\{R P(\mu \oplus(j+1) R)\}=0$.

Before this paper ends, a question is given.

QUESTION. In Proposition 3.1, if the condition that $\mu \rightarrow F$ be the fixed data of $\left(T, M^{n}\right)$ is omitted, i.e., $\mu \rightarrow F=\sqcup_{k}\left(\mu^{k} \rightarrow F^{n-k}\right)$ only be thought of as the disjoint union of some vector bundles, then does Proposition 3.1 still hold or not?

In order to answer this question, we have done many tries, but nothing conclusive. However, we conjecture that if the condition that $\mu \rightarrow F$ be the fixed data of $\left(T, M^{n}\right)$ is omitted in Proposition 3.1, then the result will still hold.

\section{REFERENCES}

[1] CONNER, P.E., Differentiable Periodic Maps, 2nd ed., Lecture Notes in Math., 738, Springer, Berlin, 1979.

[2] KOSNIOWSKI, C. and STONG, R.E., Involutions and characteristic numbers, Topology, 17 (1978), 309-330.

[3] LÜ, Z and LIU, X.B., Manifolds and involutions whose fixed point set has variable codimension, Topology and its Applications, 70 (1996), 57-65

[4] LÜ, Z. and ZHOU, C.L., Involutions fixing the disjoint union of the 3-projective space with the Dold manifold (to appear) 


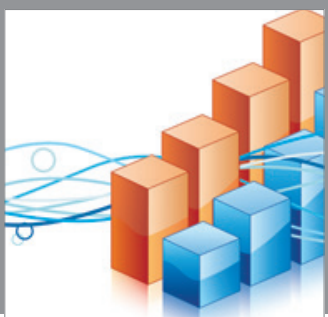

Advances in

Operations Research

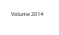

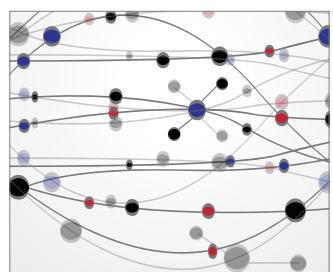

\section{The Scientific} World Journal
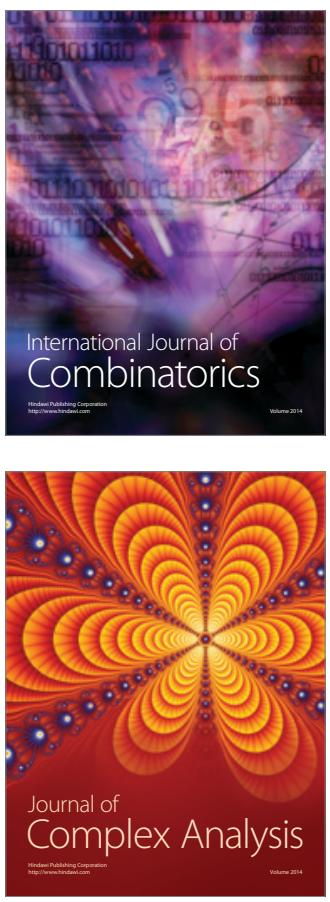

International Journal of

Mathematics and

Mathematical

Sciences
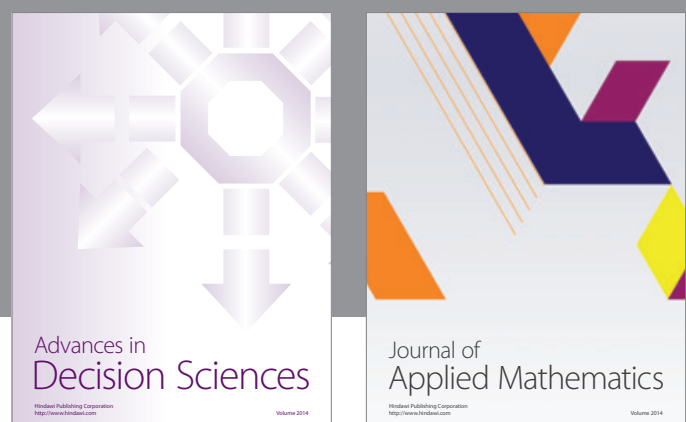

Journal of

Applied Mathematics
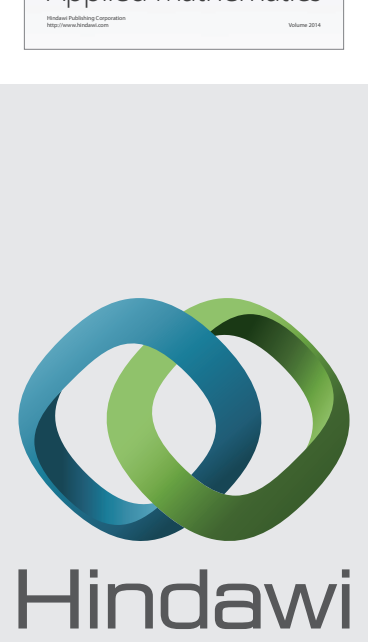

Submit your manuscripts at http://www.hindawi.com
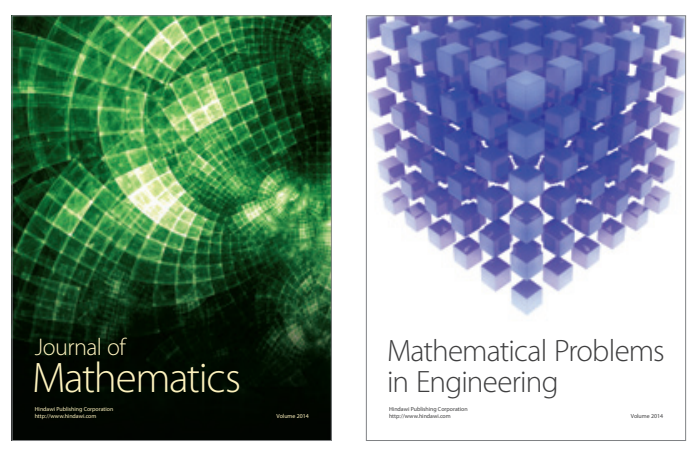

Mathematical Problems in Engineering
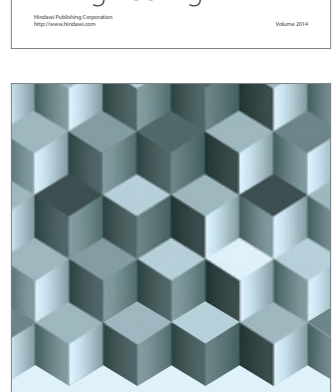

Journal of

Function Spaces
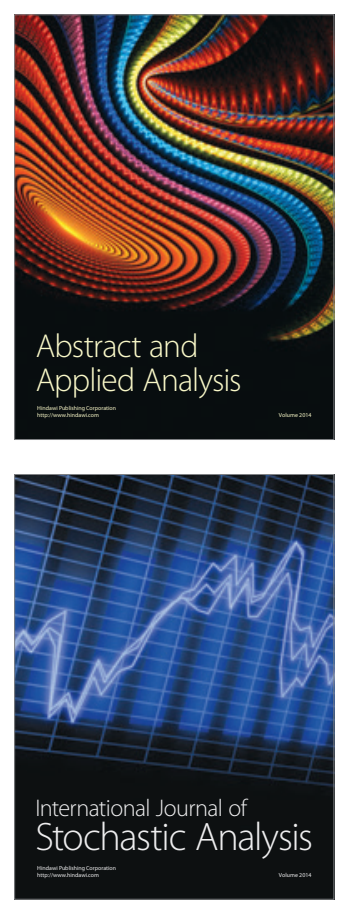

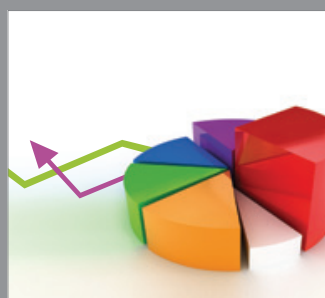

ournal of

Probability and Statistics

Promensencen
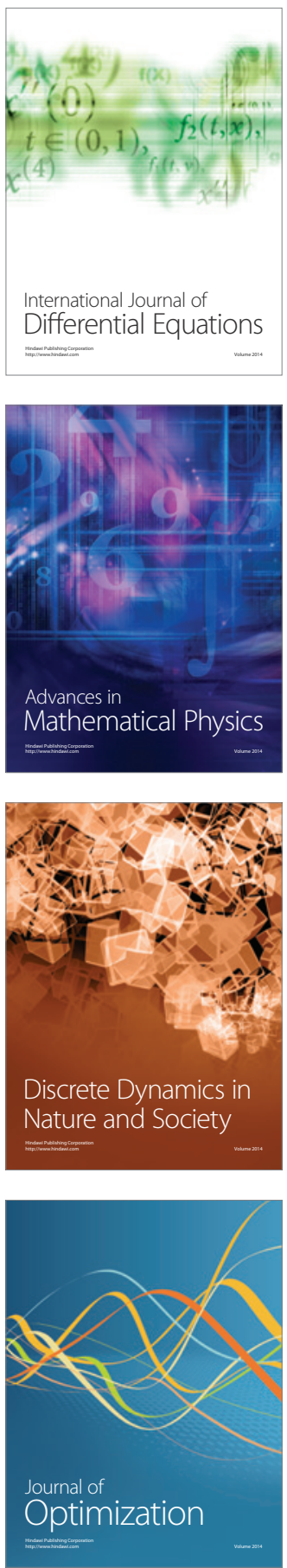